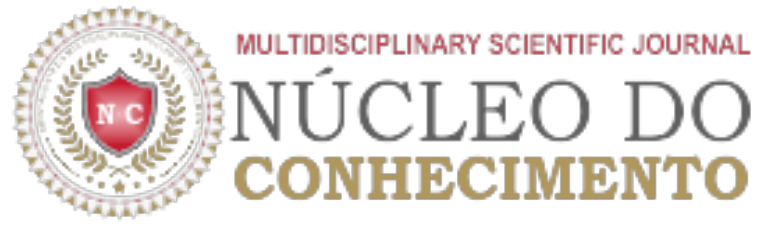

\section{Recuperação da Funcionalidade do Idoso com Gonartrose e Coxoartrose, Por meio da Hidrocinesioterapia: Um Estudo Retrospectivo}

RODRIGUES, Wanda Carla Conde ${ }^{[1]}$, ALMEIDA, Flábia Regina Da Conceição de ${ }^{[2]}$, CUSTÓDIO, Thales Odilon Marques Pena ${ }^{[3]}$, FARIAS, Luci Selma Ferreira de Freitas ${ }^{[4]}$, CARVALHO, Aline de Lima ${ }^{[5]}$,OLIVEIRA, Euzébio de ${ }^{[6]}$

RODRIGUES, Wanda Carla Conde. Et. al. Recuperação da Funcionalidade do idoso com gonartrose e coxoartrose, por meio da hidrocinesioterapia: Um estudo retrospectivo. Revista Científica Multidisciplinar Núcleo do Conhecimento. Ano 01, Ed. 07, Vol. 04, pp. 05-20, Julho de 2016. ISSN:2448-0959

\section{RESUMO}

A osteoartrose (AO) constitui em um processo degenerativo que acomete as articulações, sobretudo nas junções de suporte de peso. Com o aumento da longevidade populacional há um aumento de doenças crônicas interferindo no bem-estar. Uma das formas de tratar esta disfunção é através da hidrocinesioterapia, que pode oferecer ao idoso, qualidade de vida através da fisioterapia aquática. $\mathrm{O}$ objetivo deste trabalho foi realizar uma revisão de literatura sobre a aplicabilidade da hidrocinesioterapia e seus efeitos na funcionalidade e qualidade de vida do idoso com gonartrose e coxoartrose. Foram realizadas visitas a quatro bibliotecas de instituições de nível superior do Estado do Pará: ESAMAZ, UEPA, UFPA, UNAMA, bem como utilizadas quatro bases de dados eletrônicas: LILACS, PUBMED, SciELO, MEDLINE. Utilizou-se dos descritores: artrose, hidrocinesioterapia, coxoartrose, gonartrose, funcionalidade do idoso, juntos e separados. Foram selecionados periódicos nacionais e internacionais publicados em português e inglês. Os critérios de inclusão foram a publicação de artigos e referências no período de 1998 à 2012. A OA ocupa o terceiro lugar na lista dos segurados afastados do trabalho que recebem auxílio-doença. E de acordo com os resultados obtidos, conclui-se que a atuação fisioterapêutica através da aplicabilidade da técnica de hidrocinesioterapia é de extrema importância e benéfica na funcionalidade do idoso. Proporcionando menos dores álgicas, menos instabilidades, melhora na força muscular, melhora da flexibilidade e melhora da amplitude de movimento, facilitando então suas atividades de vida diária e melhorando sua qualidade de vida. 
Palavras-Chaves: Osteoartrose, Hidroterapia, Recuperação Funcional, Qualidade de Vida.

\section{INTRODUÇÃO}

A Osteoartrose (OA) é o resultado de vários padrões de disfunção articular, sendo caracterizada por degeneração de cartilagem articular e simultânea proliferação de tecido ósseo e de tecido conectivo. É a forma mais comum de artrite e é frequentemente associada a significante restrição e redução da qualidade de vida (QV) (FALOPPA; ALBERTONI, 2008).

Dentre as formas clínicas de OA, destacam-se a OA de quadril e joelho por serem particularmente mais incapacitantes, já que essas são as articulações que recebem todo o peso corporal. A OA de quadril atinge $20 \%$ das pessoas com idade acima de 55 anos e, apesar de ser menos comum do que a OA do joelho, sua sintomatologia é frequentemente mais grave (RICCI; COIMBRA, 2006).

Sua prevalência aumenta com a idade, de 7\% entre pessoas de 65-70 anos a 11,2\% entre aqueles com 80 anos ou mais, pouco variando entre os estudos existentes. Um recente estudo da Organização Mundial da Saúde (OMS) refere que a OA seria a quarta causa mais importante de disfunção entre mulheres e a oitava entre homens. Estudos radiográficos mostram algumas alterações em 30\% de homens e mulheres acima de 65 anos, mas apenas um terço destes são sintomáticos (RICCI; COIMBRA, 2006; DOI et al., 2008).

À medida que alguém envelhece, ocorre uma redução das funções corporais. Como por exemplo, redução da capacidade respiratória máxima e débito cardíaco. As massas muscular e óssea diminuem, enquanto a quantidade de gordura aumenta, principalmente devido a fatores endócrinos. Essa fragilidade da idade avançada é caracterizada por força muscular diminuída, reflexos lentificados, mobilidade e equilíbrio diminuídos e resistência reduzida. O resultado disso são quedas, fraturas, atividade física diária reduzida e perda da independência. A fraqueza muscular não é causada apenas por processos fisiológicos do envelhecimento e desgaste devido ao uso como em lesões articulares, mas também por falta de movimentação, levando a um ciclo vicioso (SILBERNAGL; LANG, 2006).

Com o crescimento populacional de idosos, as doenças degenerativas tornarem-se mais frequentes, trazendo consigo as disfunções causadas pelos sintomas de OA (RICCI; COIMBRA, 2006; DOI et al., 2008).

A manutenção da capacidade funcional é um dos requisitos para o envelhecimento saudável. A função física é um indicador universalmente aceito do estado de saúde e importante componente da QV. Do ponto de vista individual, a função física é necessária para manter o indivíduo independente e participante na comunidade. Dessa forma, disfunção é um problema social, que traz maior risco de institucionalização e altos custos para os serviços de saúde (TAMEGUSH et al., 2008).

Uma das formas de se tratar a disfunção é através da hidrocinesioterapia, que é capaz de abranger as necessidades de reabilitação no tratamento, além de oferecer ao idoso QV através da fisioterapia aquática (FACCI, MARQUETTI, COELHO, 2007).

A hidrocinesioterapia pode ser considerada como uma das principais intervenções terapêuticas no tratamento da OA, porém há poucos trabalhos que verifiquem seus benefícios. As propriedades físicas e 
fisiológicas da água possibilitam a realização de exercícios dificilmente executados em solo, que associados à maior amplitude de movimento (ADM) e temperatura elevada da água aumentam a mobilidade articular, o controle muscular e a resistência, aliviando dores e acelerando o processo de recuperação funcional. Ao mesmo tempo, o programa terapêutico executado na água é uma forma mais segura de fortalecimento muscular, pois não aumenta o atrito articular, como é o caso dos exercícios executados em solo (FACCI, MARQUETTI, COELHO, 2007).

Diante do exposto, a presente pesquisa teve como objetivo avaliar a recuperação da funcionalidade do idoso com gonartrose e coxoartrose, por meio da prática da hidrocinesioterapia.

\section{METODOLOGIA}

A presente pesquisa refere-se a uma análise retrospectiva realizada através de uma revisão da literatura, no período de 1998 a 2012, referente ao tema "idoso com gonartrose e coxoartrose e a recuperação da funcionalidade corporal por meio da hidrocinesioterapia. Foram realizadas visitas a quatro bibliotecas de instituições de nível superior do Estado do Pará: ESAMAZ, UEPA, UFPA, UNAMA, bem como utilizadas quatro bases de dados eletrônicas: LILACS, PUBMED, SciELO, MEDLINE. Utilizou-se para a busca mais apurada dos descritores: artrose, hidrocinesioterapia, coxoartrose, gonartrose, funcionalidade do idoso, descritos juntos e separados. Foram selecionados periódicos publicados em revistas nacionais e internacionais nos idiomas português e inglês. Os critérios de inclusão foram artigos com publicação dentro do período investigado e que atendessem ao tema e critérios do objeto da pesquisa. Foram excluídos artigos que não atendessem aos requisitos pré-determinados.

Setenta e nove artigos relacionados à pesquisa foram selecionados e analisados, dos quais trinta e quatro foram escolhidos para nortear a pesquisa, incluindo somente os que apresentaram maior relevância à mesma.

Esse método de busca permitiu encontrar artigos distintos e m,ais específicos referentes à pesquisa com relação ao objeto de estudo determinado.

\section{RESULTADOS E DISCUSSÃO}

\section{CONCEITO:}

\section{OSTEOARTROSE:}

Sinonímias

- Artrose;

- Doença articular degenerative;

- Osteoartrose;

- Osteoartrite.

A OA ou doença articular degenerativa (DAD) é caracterizada pela deterioração progressiva e ruptura da cartilagem articular, sobretudo nas articulações de suporte de peso; isso leva ao espessamento ósseo subcondral, e excrescências ósseas, osteófitos(esporões), em torno das margens das articulações. A causa é desconhecida, porém mais provavelmente relacionada com alterações metabólicas e bioquímicas 
(MITCHELL et al., 2006).

A OA constitui um processo degenerativo que acomete as articulações. As alterações começam na cartilagem articular, que sofre um processo de amolecimento e deterioração, chegando inclusive a desaparecer em fases avançadas. O osso subcondral também sofre mudanças que se traduzem em um aumento de densidade ou esclerose e na formação ocasional de cistos ou partes ocas. Nas margens articulares, aparecem prolongações ósseas do osso subcondral, de formas irregulares e tamanhos variáveis denominados de osteófitos, que constituem o sinal mais característico da OA (SACHETTI et al., 2010).

A OA é uma doença crônica e degenerativa caracterizada pela dor e pela perda gradual de cartilagem articular. A OA apresenta origem multifatorial podendo está presente em várias articulações onde ocorrem alterações bioquímicas, metabólicas e morfológicas, suas características incluem perda da configuração normal do segmento afetado, crepitação ao movimento, deformidades ósseas, formação de osteófitos, presença de processos inflamatórios e acúmulo de líquido sinovial (SILVA et al., 2011).

\section{EPIDEMIOLOGIA}

Em relação aos aspectos epidemiológicos acredita-se que cerca de $85 \%$ da população mundial apresenta evidencias radiográficas de OA por volta dos 65 anos de idade. A OA distribui-se igualmente entre homens e mulheres quando todas as idades são analisadas, no entanto quando analisamos grupos de idade superiores aos 55 anos as mulheres são mais afetadas e parecem desenvolver uma doença mais grave, provavelmente associada aos hábitos corporais ou mesmo à predisposição genética. A OA pode variar também em relação ao grupo étnico, porém essa variação pode ser mais relacionada à diferenças ocupacionais e mesmo culturais entre diversas raças. A predisposição genética envolve principalmente as apresentações nodais de OA de mãos e algumas de OA primária (FREITAS; PY, 2011).

\section{ETIOLOGIA}

Para Carvalho e Papaléo (2006) a OA é de causa não definida, e pode ser classificada em diversas formas:

- Forma primária (idiopática): existe uma degeneração cartilaginosa na articulação aparentemente normal sem causa evidente.

- Forma secundária: a degeneração cartilaginosa é consequiência de alterações articulares preexistentes.

Diversos fatores podem estar envolvidos na etiologia da OA, como: idade, predisposição genética, traumas, estresse repetitivo, algumas ocupações, obesidade, alterações na morfologia da articulação, instabilidade articular e alterações na bioquímica da cartilagem articular (CARVALHO; PAPALÉO, 2006).

Segundo Paradiso (1998) a OA pode ter influências mecânicas e hereditárias e podem ocorrer secundariamente à outra doença articular, tal como dano traumático ou de esporte. A OA que afeta comumente o quadril e os joelhos, são as formas mais amplamente ocorrente de doença articular.

\section{FISIOPATOLOGIA}


$\mathrm{Na}$ cartilagem articular as mudanças nos condrócitos e na matriz cartilaginosa resultam na perda da estrutura normal. Com a progressão da doença, algumas partes da superfície articular se tornam mais irregulares e a fibrilação se estende para as camadas mais profundas da cartilagem, ultrapassando a zona intermediária e em seguida alcançando o osso subcondral. Quando as fissuras avançam mais profundamente, as extremidades superficiais da cartilagem fibrilada se desgastam, diminuindo a espessura da cartilagem e liberando fragmentos soltos no espaço articular. Ao mesmo tempo, a degradação enzimática da matriz pode diminuir ainda mais o volume da cartilagem. Em estados avançados, o osso subcondral pode tornar-se exposto (Figura 1) (REBELATTO; MORELLI, 2007). 


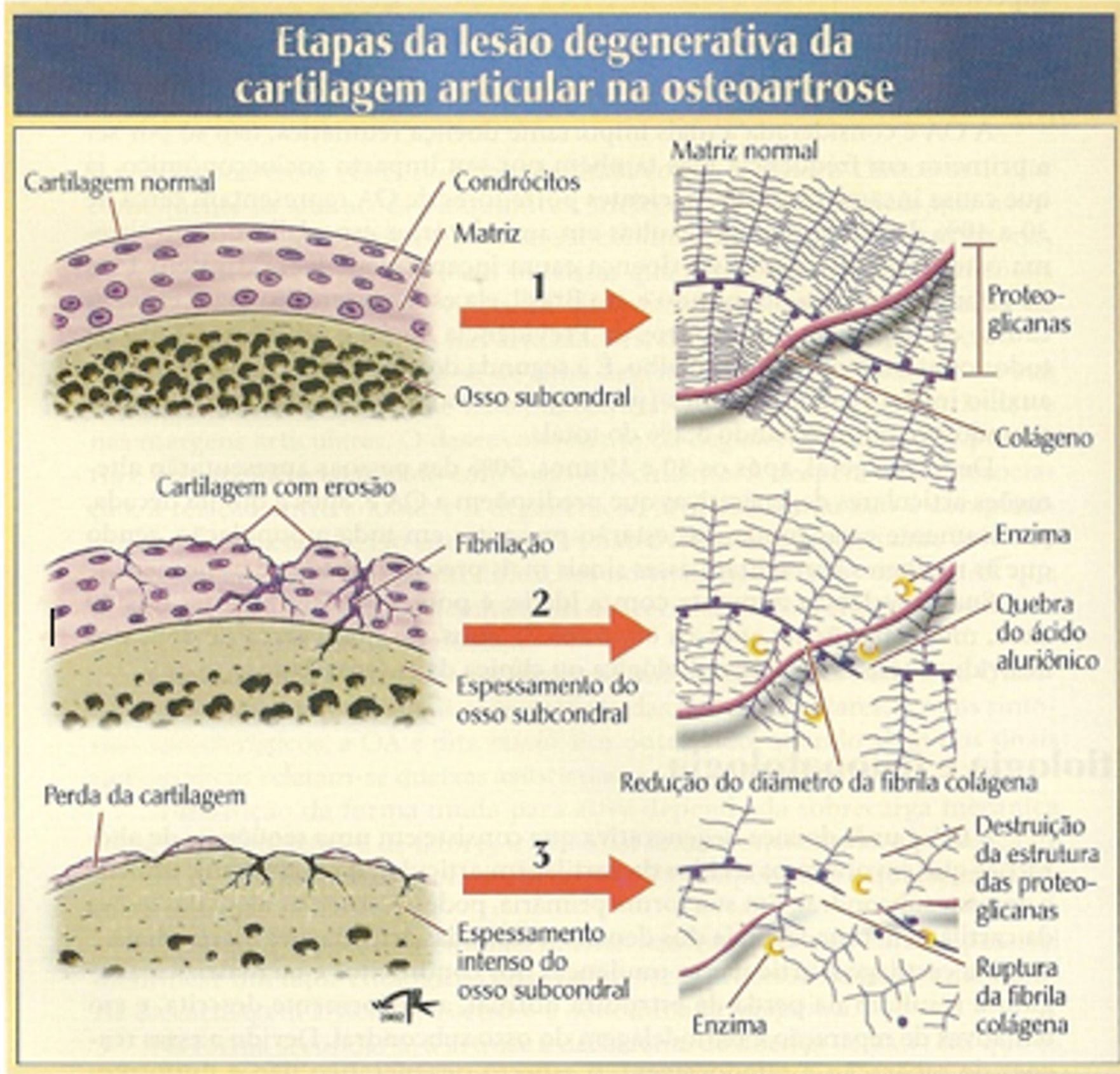

Figura 1: Fisiopatologia da Osteoartrose.

Fonte: REBELATTO; MORELLI, 2007.

Em decorrência do processo patológico característico da OA, observa-se a degradação cartilaginosa, acompanhada de alterações ósseas representadas por esclerose ou eburnificação do osso subcondral e por remodelagem articular com a participação, muitas vezes de reação inflamatória sinovial (REBELATTO; MORELLI, 2007).

\section{CLASSIFICAÇÃO}

A classificação proposta por Kellgren-Lawrence (1979), tem sido a mais amplamente utilizada na literatura até hoje, embora novas tecnologias de diagnóstico por imagem, como a radiografia microfocal, 
a ressonância magnética $(\mathrm{RM})$ e a ultrassonografia articular, sejam mais precisas (NATALIO; OLIVEIRA; MACHADO, 2010).

Os critérios definidos por Kellgren-Lawrence vão de I à IV e são classificados como:

- Grau I: provável diminuição do espaço articular, com possível osteófitose;

- Grau II: osteófitos bem definidos e possível diminuição do espaço articular;

- Grau III: múltiplos osteófitos, clara diminuição do espaço articular e possíveis deformidades nas extremidades ósseas;

- Grau IV: grandes osteófitos, intensa diminuição do espaço articular, esclerose grave e extremidades ósseas com deformidades definidas (VASCONCELOS; DIAS; DIAS, 2006).

\section{SINAIS E SINTOMAS}

Clinicamente a OA manifesta-se por dor que varia de leve a muito intensa, que piora com os movimentos e ao levantar peso, rigidez articular que se agrava pelo repouso, limitação de movimentos e crepitação (PORTO, 2008).

Para Merklee (2007) os sinais e sintomas da AO aumentam com a má postura, obesidade e estresse ocupacional podendo incluir:

- Dor articular profunda provocada por degradação da cartilagem, inflamação e estresse ósseo, em particular após exercícios ou sustentar peso, é o sintoma mais comum, na maior parte das vezes, é aliviado pelo repouso.

- Rigidez pela manhã e após prática de exercícios (aliviada pelo repouso), suas causas são dadas pela degeneração da cartilagem, inflamação e estresse ósseo.

- Crepitação ou 'raspar' da cartilagem durante a movimentação decorrente de lesão da cartilagem.

- Nódulos de Heberden (alargamentos ósseos das articulações interfalângicas distais), originados por inflamação repetida (Figura 2). 


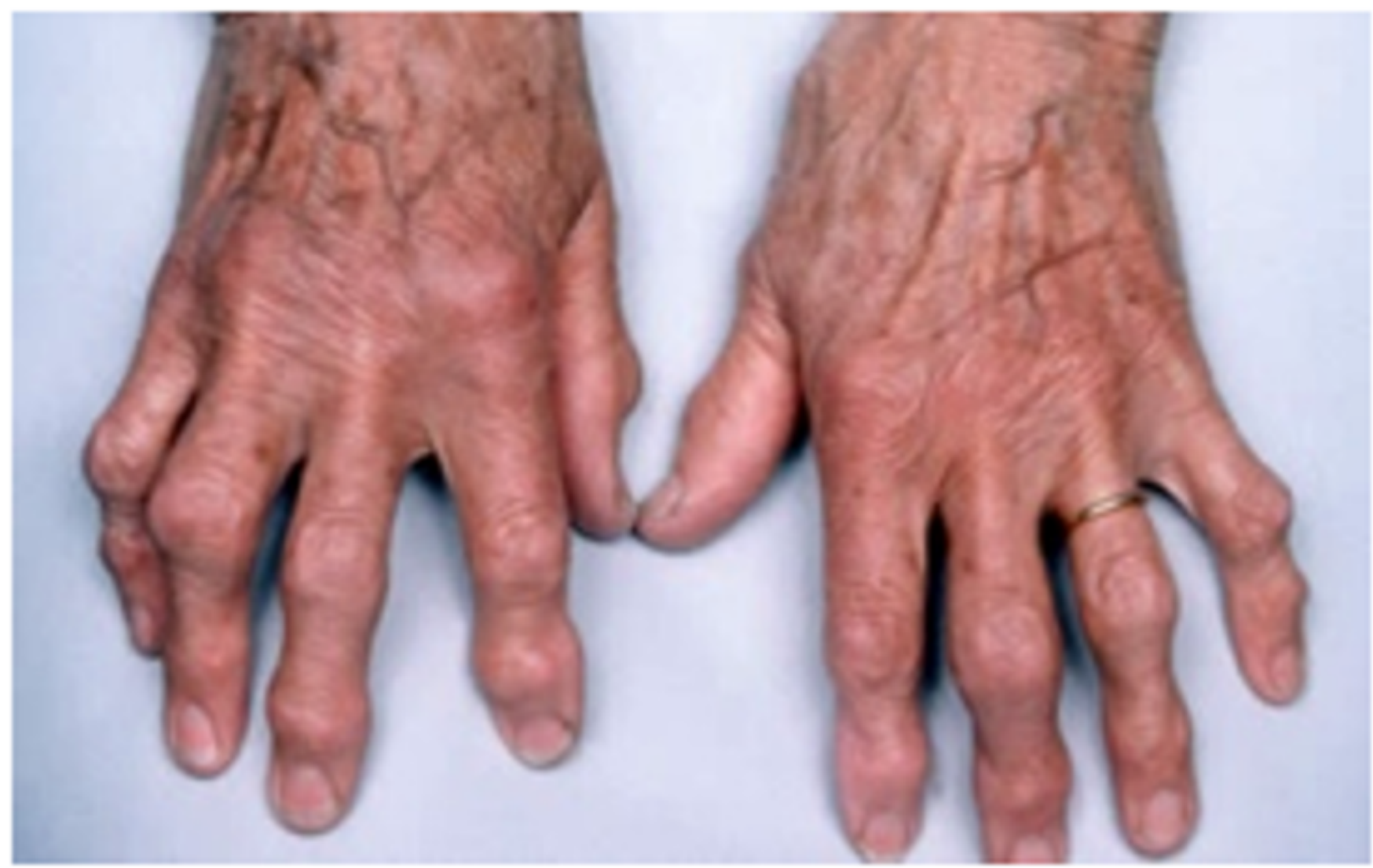

Figura 2: Nódulos de Heberden e Bouchard Fonte: www.portalsaofrancisco.com.br/alfa/reumatismo/

- Alteração da marcha em virtude de contraturas dos músculos que sustentam a articulação.

- Diminuição da ADM em razão da dor e rigidez articular.

- Alargamento da articulação, induzido por estresse sobre o osso e crescimento ósseo desordenado.

\section{HIDROTERAPIA}

A hidroterapia é toda aplicação externa de água, em qualquer de seus estados físicos, com finalidade terapêutica (DEGANI, 1998).

A hidroterapia é um recurso muito utilizado na reabilitação de pacientes com OA que possuem manifestações articulares e extra-articulares devido às propriedades físicas e efeitos fisiológicos da água (FERREIRA et al., 2008).

Com isso a fisioterapia faz uso deste recurso por obter um grupo de possibilidades de intervenções clínicas através da cinesioterapia em piscinas aquecidas, como recurso auxiliar da reabilitação ou prevenção de alterações funcionais. As propriedades físicas e o aquecimento da água desempenham um papel importante na melhoria, manutenção da ADM das articulações, redução da tensão muscular e no relaxamento (CANDELORO; CAROMANO, 2007).

Sendo um dos recursos mais antigos da fisioterapia, a hidroterapia é definida como o uso externo da água com propósitos terapêuticos. É um recurso muito utilizado no processo de reabilitação especialmente em pacientes portadores de $\mathrm{OA}$, por possuir algumas vantagens devido às propriedades físicas e efeitos 
fisiológicos, proporcionado pelo meio aquático (RUOTI; MORRIS; COLE, 2000).

A hidroterapia é frequentemente recomendada para pacientes com OA, pois proporciona uma gama de benefícios incluindo redução de edema, da dor e da sobrecarga sobre as articulações já lesionadas (FOLEY ET al., 2003).

A hidroterapia promove reações diferentes daquelas experimentadas em solo, melhorando a circulação periférica, beneficiando o retorno venoso, além de proporcionar um efeito massageador e relaxante ao paciente, atuando dessa forma nas principais queixas de pacientes com OA. Os exercícios na água são muito bem tolerados, especialmente em água aquecida, pois o ambiente morno ajuda a reduzir a dor e espasmos musculares (REILLY; BIRD, 2001).

A água oferece suave resistência durante os movimentos e, ainda, a oportunidade de treinamento em várias velocidades. Esses componentes fazem com que o exercício aquático seja um excelente método para aumento da resistência e força muscular (CAMPION, 2000).

\section{PROPRIEDADES DA ÁGUA}

O entendimento dos princípios físicos e da termodinâmica da água proporciona o seu uso racional (BIAZUS; PADILHA; LOUREIRO, 2010).

A flutuação permite ao paciente caminhar com pouca sobrecarga articular devido à redução dos efeitos da gravidade e ao consequente aumento da ADM articular (KISNER; COLBY, 2005).

A pressão hidrostática exerce um efeito positivo, durante a imersão (a mesma pressão é exercida em todas as direções do corpo e aumenta com a profundidade), de diminuição de edemas, pois o sistema venoso redistribui o sangue das extremidades para o tórax (KISNER; COLBY, 2005).

A resistência da água pode ser utilizada passiva e ativamente, movimentos passivos através da água facilitam o relaxamento e alongamento de tecidos moles, ativamente a resistência da água aumenta o gasto energético necessário para movimentar as extremidades, promovendo fortalecimento muscular (BUENO et al., 2007).

A termodinâmica também deve ser levada em consideração e está relacionada com a capacidade de o corpo submerso trocar energia (calor) com a água por meio da condução e convecção, tendo efeito positivo sobre tecidos moles que podem facilmente ser mobilizados, aumentando o grau de movimento e diminuindo a dor articular (SACCHELLI; ACCACIO; RADL, 2007).

Os benefícios proporcionados pela água são: relaxamento muscular, aumento do fluxo sanguíneo e da flexibilidade, fortalecimento muscular, reeducação da marcha, melhora do equilíbrio e da coordenação e, finalmente, é uma atividade lúdica e de recreação (BUENO et al., 2007).

\section{EFEITOS FISIOLÓGICOS}

Os efeitos fisiológicos da hidroterapia advêm de uma combinação dos efeitos físicos da água (térmicos e mecânicos) e efeitos do exercício. Os efeitos variam com a duração do tratamento, exercício, tipo, 
progressão, intensidade do exercício, temperatura da água, postura, movimentos associados dos membros superiores e também da patologia do paciente (IDE et al., 2004).

Com os efeitos da imersão em água aquecida ocorrem também a elevação da temperatura corpórea mesmo no repouso, desde que a temperatura da água esteja mais elevada que a da pele, aproximadamente $35,5^{\circ} \mathrm{C}$. O aumento na temperatura corpórea também ocorre pela conversão de energia durante o exercício. Depende da intensidade do exercício e também da quantidade de gordura corporal do paciente (RUOTI; MORRIS; COLE, 2000).

\section{HIDROCINESIOTERAPIA}

A hidrocinesioterapia incorporou os recentes avanços do conhecimento de avaliação físico-funcional, prática baseada em evidências, experiência clínica aos princípios da hidrostática, hidrodinâmica e fisiologia de imersão, para traçar e embasar os planos de tratamentos dos pacientes na piscina terapêutica (GOMES, DIAS, CISNEROS, 2007).

Os exercícios aquáticos têm sido largamente utilizados nos programas de fisioterapia, especialmente quando os exercícios nos solo geram dor. A diminuição do impacto articular durante atividades físicas induzida pela flutuação, causa redução da sensibilidade à dor, diminuição da compressão nas articulações doloridas, maior liberdade de movimento e diminuição do espasmo doloroso. O efeito de flutuação auxilia o movimento das articulações rígidas em amplitudes maiores com um aumento mínimo de dor, permitindo a realização de exercícios que no solo poderiam ser considerados muito difíceis (CANDELORO; CAROMANO, 2007), (HINMAN; HEYWOOD; ANTHONY, 2006), (SILVA et al, 2011).

Os exercícios de fortalecimento com paciente submerso estão fundamentados nos princípios físicos da hidrostática, que permitem gerar resistência multidimensional constante aos movimentos. Essa resistência aumenta proporcionalmente à medida que a força é exercida contra ela, gerando uma sobrecarga mínima nas articulações (CANDELORO; CAROMANO, 2007).

Um programa abrangente de hidrocinesioterapia satisfaz as necessidades da reabilitação e de condicionamento físico do paciente, levando em consideração os componentes psicológicos, ?siológicos e sociológicos da QV. Dentro deste contexto, a promoção e a atenção à saúde do idoso englobam medidas preventivas, restauradoras e reabilitativas, priorizando preservar, manter, restaurar ou desenvolver a função para o paciente portador de coxoartrose e gonartrose (KOURY, 2000).

\section{INDICAÇÕES}

Segundo Koury (2000) a hidroterapia é indicada quando se deseja pouca ou nenhuma sustentação de peso, ou quando há processo inflamatório agudo, dor, retração e espasmo muscular e limitação da ADM, que podem de maneira isolada ou conjunta diminuir a função normal. A hidroterapia também é uma opção para pacientes que estejam incapacitados de realizar exercícios no solo em razão de cirurgia recente, lesão neuromuscular ou ortopédica aguda, doença reumatológica ou deficiência neurológica.

A indicação da hidrocinesioterapia em pacientes com $\mathrm{OA}$ visa manter a mobilidade articular, o alongamento da musculatura e aumentar a ADM, melhorando a estabilidade articular. Além disso, tem 
como objetivo otimizar a biomecânica articular para manter o alinhamento correto do segmento afetado e reduzir qualquer excesso de carga anormal sobre a articulação envolvida. O alívio da dor, da rigidez e de outros sintomas associados também faz parte dos objetivos da reabilitação física (IZOLA; BIASOLI, 2003).

\section{CONTRA-INDICAÇÕES}

Segundo Kisner e Kolby (2005) são contra-indicações:

- Insuficiência cardíaca incipiente e angina instável.

- Disfunção respiratória, capacidade vital abaixo de 1 litro.

- Doença vascular periférica grave.

- Risco de sangramento ou hemorragia.

- Doença renal grave: os pacientes serão incapazes de ajustar a perda de líquido durante a imersão.

- Feridas abertas, colostomia e infecções de pele.

- Incontinência urinária e fecal.

- Infecções ou doenças transmissíveis pela água e pelo ar: exemplos incluem influenza, infecções gastrintestinais, tifo, cólera e poliomielite.

- Convulsões descontroladas: elas criam um problema de segurança, tanto para o profissional quanto para o paciente, caso seja necessária a remoção imediata da piscina.

Os mesmos autores informam que o propósito específico do exercício aquático é facilitar a recuperação funcional, proporcionando um ambiente que aumente a habilidade do paciente e/ou do profissional de realizar diferentes intervenções fisioterapêuticas, que visam:

- Facilitar os exercícios de ADM;

- Iniciar o treinamento resistido;

- Facilitar atividades com descarga de peso;

- Favorecer a aplicação de técnicas manuais;

- Promover o acesso tridimensional ao paciente;

- Facilitar os exercícios cardiovasculares;

- Iniciar a simulação de atividades funcionais;

- Minimizar a lesão e diminuir os riscos de lesão recidivante durante a reabilitação;

- Favorecer o relaxamento do paciente.

\section{CONSIDERAÇÕES FINAIS}

De acordo com os artigos analisados na presente pesquisa, é possível observar que a atuação fisioterapêutica através da técnica de hidrocinesioterapia é de extrema importância e na recuperação e/ou na funcionalidade e QV do idoso com gonartrose e coxoartrose. Sendo hoje uma das técnicas terapêuticas de maior destaque para o tratamento da sintomatologia dessas doenças.

Desta forma, com este estudo de revisão bibliográfica espera-se a contribuição para a literatura e para a sociedade de maneira geral incentivando os pesquisadores e profissionais atuantes na área, a buscarem evidências científicas sobre o assunto aprimorando a sua aplicabilidade para que ela seja melhor desenvolvida junto aos respectivos pacientes. 
Sendo assim, ao finalizar este estudo, pode-se concluir que a aplicabilidade da hidrocinesioterapia é benéfica e muito eficaz no tratamento de pacientes idosos portadores de gonartrose e coxoartrose, desde que sua técnica seja aplicada de forma correta e/ou com o auxílio de outras técnicas e métodos de tratamento. Seus efeitos proporcionam melhoras significativas da funcionalidade do indivíduo idoso proporcionando assim, maior QV ao mesmo. Contudo esperamos que esse método terapêutico proporcione ao idoso uma condição em que ele se sinta mais seguro e confiante tendo independência e autonomia em deu dia-a-dia para a realização de suas atividades de vida diária (AVD’S).

\section{REFERÊNCIAS}

BIAZUS J, F; PADILHA J, F; LOUREIRO L, F. Terapia aquática na artrite reumatóide juvenil: relato de caso. Promovendo Saúde na Contemporaneidade: desafios de pesquisa, ensino e extensão Santa Maria, RS, 08 a 11 de junho de 2010. Disponível em <http://www.unifra.br/eventos/jis2010/Trabalhos/301.pdf>. Acesso em 04/10/2012.

BUENO, V.C.; LOMBARDI JÚNIOR, I.; MEDEIROS, W.M.; AZEVEDO, M.M.A.; LEN, C.A.; TERRERI, M.T.R.A.; NATOUR, J.; HILÁRIO, M.O.E. Reabilitação em Artrite Idiopática Juvenil Rehabilitation in Juvenile Idiopathic Arthritis. Revista Brasileira de Reumatologia, v. 47, n.3, p. 197-203, mai/jun, 2007. Disponível em < http://www.scielo.br/pdf/rbr/v47n3/09.pdf>. Acesso em $08 / 08 / 2012$.

CAMPION, M.R. Hidroterapia: Princípios e Prática. São Paulo: Manole, 2000.

CANDELORO, J.M.; CAROMANO, F.A. Efeito de um programa de hidroterapia na flexibilidade e na força muscular de idosas. Revista Brasileira de Fisioterapia, São Carlos, v. 11, n. 4, p. 303-309, jul./ago. 2007.

CARVALHO FILHO, E.T.; PAPALÉO NETTO, M.P. Geriatria Fundamentos, Clínica e Terapêutica. $2^{\text {a }}$ edição. São Paulo: Atheneu, 2006.

DEGANI, A.M. Hidroterapia: os efeitos físicos, fisiológicos e terapêuticos da água. Fisioterapia em Movimento. Curitiba: Champagnat, v. XI, n. 1, abr./set. 1998.

DOI T, AKAI M, FUJINO K, IWAYA T, KUROSAWA H, HAYASHI K, et al. Effect of home exercise of quadriceps on knee osteoarthritis compared with nonsteroidal antiinflammatory drugs: a randomized controlled trial. Am J Phys Med Rehabil. 2008;87(4):258-69. doi:10.1097/PHM.0b013e318168c02d.

FACCI, L.M.; MARQUETTI, R.; COELHO, K.C. Fisioterapia aquática no tratamento da Osteoartrite de joelho: série de casos. Fisioterapia em Movimento, Curitiba, v. 20, n. 1, p. 17-27, jan./mar., 2007.

FALOPPA, F.; ALBERTONI, W. M. Guias de medicina ambulatorial e hospitalar da UNIFESPEPM. $1^{a}$ edição. São Paulo: Manole: 2008.

FERREIRA, L.R.F.; PESTANA, P.R.; OLIVEIRA, J. ; FERRARI, R.A.M. Efeitos da reabilitação aquática na sintomatologia e qualidade de vida de portadoras de artrite reumatoide. Fisioterapia e Pesquisa, São Paulo, v.15, n.2, p.136-41, abr./jun. 2008. 
FOLEY, A.; HALBERT, J.; HEWITT, T.; CROTTY, M. Does hydrotherapy improve strength and physical function in patients with osteoarthritis- a randomised controlled trial comparing a gym based and a hydrotherapy based strengthening programme. Ann Rheumathology Disease, 2003; 62:1162-1167. doi: 10.1136/ard.2002.005272. Disponível em <http://ard.bmj.com/content/62/12/1162.full.pdf+html>. Acesso em 13/08/2012.

FREITAS, E.V.; PY, L. Tratado de Geriatria e Gerontologia. $3^{\text {a }}$ edição. Rio de Janeiro: Guanabara Koogan, 2011.

GOMES, W.F.; DIAS, J.M.D.; CISNEROS, L.L. Impacto de um programa estruturado de fisioterapia aquática em idosas com osteoartrite de joelho. 2007. Dissertação (Mestrado em Fisioterapia) - Universidade Federal de Minas Gerais, Belo Horizonte, 2007.

HINMAN, R.S.; HEYWOOD, S. E.; ANTHONY, R. Day Osteoarthritis: Results of a Single-Blind Randomized Aquatic Physical Therapy for Hip and Knee Controlled Trial doi: 10.2522/ptj.20060006 Originally published online January 1, 2006. PHYS THERAPY. 2007; 87:32-4. Disponível em http://physther.org/content/87/1/32.full.pdf+html . Acesso em 14/08/2012.

IDE, M.R.; YNOUE, A.T.; FARIAS, N.C.F.; CHÃO, C.C.; ROSA, A.R. Fisioterapia Aquática nas Disfunções do Aparelho Locomotor. Anais do $2^{\circ}$ Congresso Brasileiro de Extensão Universitária Belo Horizonte - 12 a 15 de setembro de 2004.

IZOLA, L.N.T.; BIASOLI, M.C. Aspectos gerais da reabilitação física em pacientes com osteoartrose. RBM - Revista Brasileira de Medicina, - vol. 60 - nº 3 - março de 2003.

KELLGREN, J.H. Osteoarthrisis in Patients and Populations. Br Med Journal, 1979; 2:1-6.

KISNER, C.; COLBY, L.A. Exercício terapêutico fundamentos e técnicas. $4^{\mathrm{a}}$ edição. São Paulo: Manole, 2005.

KOURY, J. Programa de ?sioterapia aquática. São Paulo: Manole, 2000.

MERKLEE, C.J. Manual de fisiopatologia. 2a edição. São Paulo: Rooca, 2007.

MITCHELL, R. N,; KUMAR, V,; ABBAS, A. K.; FAUSTO, N. Fundamentos de patologia bases patológicas das doenças. Rio de Janeiro: Elsevier, 2006.

NATALIO, M.A.; OLIVEIRA, R.B.C.; MACHADO, L.V.H. Osteoartrose: uma revisão de literatura. Revista Digital, Buenos Aires, año 15 - n. 146 - julio de 2010.

PARADISO, C. Fisiopatologia série de estudos em enfermagem. Rio de Janeiro: Guanabara Koogan, 1998.

PORTO, C.C. Exame clínico bases para a prática médica. $6^{a}$ edição. Rio de Janeiro: Guanabara Koogan, 2008. 
REBELATTO, J.R.; MORELLI, J.G.S. Fisioterapia Geriátrica. 2a edição. São Paulo: Manole, 2007.

REILLY, K.A.; BIRD, H.A. Prophylactic hydrotherapy. Brasilian Society Rheumatology. 2001;40:4-6. Disponível em < http://www.scielo.br/scielo.php?pid=S1809-2950200800020 0005\&script=sci_arttext >. Acesso em 10/08/2012.

RICCI, R.; COIMBRA, I. Exercício Físico como Tratamento na Osteoartrite de Quadril: uma Revisão de Ensaios Clínicos Aleatórios Controlados, 2006. Disponível em <http://www.scielo.br/pdf/rbr/v46n4/31823.pdf>. Acesso em 02/02/2012.

RUOTI, R.G.; MORRIS, D.M.; COLE, A.J. Reabilitação aquática. 1ª edição. São Paulo: Manole, 2000.

SACCHELLI, T.; ACCACIO, L.M.P.; RADI, A.L.M. Manual de fisioterapia aquática. $1^{\text {a }}$ edição. São Paulo: Manole, 2007.

SACHETTI, A.; VIDMAR, M.F.; VENÂNCIO, G.; TOMBINI, D.K.; SORDI, S.; PILLA, S.; SILVEIRA, M.M.; WIBELINGER, L.M. Perfil Epidemiológico de Idosos com Osteoartrose. Revista de Ciências Médicas e Biológicas, Vol. 9, n. ${ }^{\circ}$ 3, 2010.

SILBERNAGL, S.; LANG, F. Fisiopatologia texto e atlas. $1^{\text {a }}$ edição. Porto Alegre: Artmed, 2006.

SILVA, A.; SERRÃO, P.R.M.S.; DRIUSSO, P.; MATTIELLO, S.M. Efeitos de exercícios terapêuticos no equilíbrio de mulheres com Osteoartrite de Joelho: Uma revisão sistemática. Revista Brasileira de Fisioterapia, [online]. ahead of print, pp. 0-0. Epub Dec 22, 2011. ISSN 1413-3555. Disponível em <http://dx.doi.org/10.1590/S1413-35552011005000035. 2011>. Acesso em 02/08/2012.

TAMEGUSH, A.; TRElHA, C.; DELlarOZA, M.; CABRERA, M.; RIBEIRO, T. Capacidade Funcional de Idosos com Osteoartrite de Joelhos e Quadril. Revista Espaço para a Saúde, Londrina, v.9, n.2, p.08-16, jun.2008.

VASCONCELOS, K.S.S.; DIAS, J.M.D.; DIAS, R.C.R. Relação entre intensidade dor e capacidade funcional em indivíduos obesos com osteoartrite de joelho. Revista Brasileira de Fisioterapia, Belo Horizonte, v.10, n.2, p.213-218, 2006.

[1] Mestre em Motricidade Humana, Especialista em Traumato Ortopedia, Docente da Faculdade Metropolitana da Amazônia.

${ }^{[2]}$ Acadêmica de Fisioterapia pela ESAMAZ - Escola Superior da Amazônia.

[3] Acadêmico de Fisioterapia pela ESAMAZ - Escola Superior da Amazônia.

${ }^{[4]}$ Acadêmica de Enfermagem pela FAMAZ - Faculdade Metropolitana da Amazônia.

[5] Acadêmica de Enfermagem pela FAMAZ - Faculdade Metropolitana da Amazônia. 
${ }^{[6]}$ Biólogo. Doutor em Medicina/Doenças Tropicais, Docente e Pesquisador pela UFPA, Pesquisador do Programa de Pós Graduação do Núcleo de Medicina Tropical da UFPA.

\section{PUBLIQUE SEU ARTIGO CIENTÍFICO EM:}

https://www.nucleodoconhecimento.com.br/enviar-artigo-cientifico-para-submissao 